\title{
Selective orienting and habituation of the GSR as a consequence of overt and covert activity
}

\author{
IRVING MALTZMAN and DAVID C. RASKIN \\ University of California, Los Angeles, Los Angeles, California 90024
}

\begin{abstract}
Effects of instructions as predeterminers of the OR were studied during habituation of the GSR to innocuous words. Different groups of college students were instructed to overtly respond to a particular word, covertly free-associate or count to that word, or simply listen. Significantly greater responsivitiy to the signal word was displayed by the three experimental groups than by the control group. Magnitude of responsivity to the unrelated filler words did not differ among the four groups. Instructions had similar effects on responsivity to all words in a habituation phase prior to the occurrence of the signal word. Experimental groups receiving task instructions, however, did not differ significantly from the control group in the magnitude of their GSR-OR to the first word in the habituation series.
\end{abstract}

The present experiment was stimulated by the studies of the OR reported by Luria and Vinogradova (1959). They reported that, with normal children as participants, attaching signal value to a word by instructing the children to perform a motor response upon its appearance retarded habituation of the digital vasomotor index of the OR to that word. Significance imparted to the word in such a manner also resulted in semantic generalization to related words in the absence of the motor response.

The purpose of the present experiment was to extend the original Luria and Vinogradova studies by determining whether implicit activity in relation to a particular word would also enhance measures of the OR to that word. Four groups of college students were employed for this purpose. One group was instructed to free-associate to themselves whenever they heard a particular word. A second group was instructed to count to themselves the number of times a particular word occurred. A third group, corresponding to the Luria and Vinogradova procedure, was instructed to press a foot pedal upon the presentation of a particular word. A control group was also employed which was instructed to sit quietly and listen to a series of words that would be presented to them.

The research reported here was made possible in part by funds provided by the Carnegie Corporation of New York and from Public Health Service Research Grant MH 04684 from the National Institute of Mental Health. For the analyses of experimental results reported here, computing assistance was obtained from the Health Science Computing Facility, UCLA, sponsored by National Institutes of Health Grant FR-3. We are indebted to O. J. Barnett and C. Wolff for their assistance. Portions of the results from this experiment have been described elsewhere (Maltzman, 1971; Maltzman \& Raskin, 1965). David C. Raskin is now at the University of Utah. Requests for reprints should be sent to Irving Maltzman, Department of Psychology, University of California, Los Angeles, California 90024.

\section{METHOD}

\section{Subjects}

There were 140 University of California, Los Angeles students who participated in the experiment for partial fulfillment of a course requirement. Approximately an equal number of men and women served in each of the four groups.

\section{Procedure}

Students in the associate group ( $\mathrm{n}=36)$ were asked to implicitly associate to the word "light" each time that they heard it. Students in the pedal-press group $(n=34)$ were instructed to press a foot pedal every time they heard "light." Students in the count group $(n=36)$ were asked to implicitly count the number of times that they heard "light," and the control group $(n=34)$ was asked to simply listen to the words.

Twenty different neutral words constituting a habituation series was initially presented. A training session followed consisting of 14 presentations of "light" interspersed among 24 neutral filler words which were repeated in three different random orders. From two to seven filler words intervened between successive presentations of "light." The interval between successive words varied from 8 to $12 \mathrm{sec}$. A generalization test, consisting of a single presentation of the words "lamp," "dark," "heavy,", "soft," "square," and "light," followed the training phase without interruption. Following Mednick (1957), the generalization test words were selected because they varied systematically in associative strength with the training word "light," as determined from word association norms (Russell \& Jenkins, Note 1).

We now know that order effects in generalization can be extremely important (Maltzman \& Langdon, 1969). Since only a single order of presentation was employed with all students, the results from the generalization test are confounded by an order effect and therefore will not be presented. All filler words employed in the experiment were selected on the basis of word association norms (Russell \& Jenkins, Note 1) which indicated that they were not associates of either the training word "light" or any of the generalization test words. There was a single presentation of a 1 -sec 90-dB (A) noise $20 \mathrm{sec}$ after the presentation of the last word. All stimuli were prerecorded on magnetic tape and presented via stereophonic headphones.

\section{Apparatus}

The experimental setting has been previously described (Maltzman, Gould, Barnett, Raskin, \& Wolff, 1977). Recording of the GSR was accomplished by means of palmar GSR electrodes consisting of two 20 -mm zinc disk electrodes fitted in 5-mm-deep 
plastic cups. The cups were filled with electrode paste made from agar and a $1 \%$ zinc sulfate solution and were attached to the palms by plastic pressure clips. The GSR was recorded using a 40- $\mu \mathrm{A}$ Wheatstone bridge and one channel of a Sanborn dual dc amplifier-recorder.

The GSR was scored as the largest resistance change occurring within the interval $.5-5.5 \mathrm{sec}$ following each of the words during habituation, the critical word "light" and the immediately preceding $\left(C_{1}\right)$ and following $\left(C_{2}\right)$ control words, as well as the final noise presentation. Log conductance base level at the time each stimulus was presented was also determined. Prior to statistical analyses, the GSR was subjected to a log conductance change transformation.

The .05 confidence level for a two-tailed test was employed in all statistical analyses.

\section{RESULTS AND DISCUSSION}

All students were categorized as high- or low-OR participants in terms of whether they were above or below the median of the distribution of GSR magnitudes evoked by the first word presented in the habituation session.

\section{Habituation.}

Mean GSR magnitudes over the 20 -word habituation session for the associate, pedal-press, count, and control groups were $1.10,1.24, .87$, and .75 , respectively.

Anaiysis of variance yielded a significant effect for Groups $[F(3,132)=6.07]$ and OR level $[F(1,132)$ $\left.=67.69, \mathrm{MS}_{\mathrm{B}}=5.430\right]$. As to be expected, Trials was also significant $[\mathrm{F}(19,2508)=65.14, \mathrm{MSW}=$ .291]. Analyses of orthogonal polynomials indicated that the linear components of the trends obtained by the different groups differed significantly $[\mathrm{F}(3,132)$ $\left.=3.52, \mathrm{MS}_{\mathrm{B}}=669\right]$. The quadratic components of the trends also differed $\left[\mathrm{F}(3,132)=4.73, \mathrm{MSB}_{\mathrm{B}}=\right.$ .382]. Individual comparisons of the overall mean GSR magnitudes produced by the four groups during habituation by Dunnett's test, and t tests yielded the same results. The associate and pedal-press groups obtained significantly larger mean GSRs than the control group, while the count group did not. The pedal-press group also obtained significantly larger GSRs than the count group. No other differences were significant.

The significant effect yielded by OR level is not surprising. This between-subjects effect is repeatedly obtained (e.g., Maltzman, Langdon, Pendery, \& Wolff, 1977), especially in habituation where the selection of high- and low-OR groups is made on the basis of their response to the first word in habituation. But another characteristic of the GSR-OR, the absence of an initial difference among the various groups, is of considerable interest. Mean magnitudes of the GSR evoked by the first word in the habituation session for the associate, pedal-press, count, and control groups were $2.16,2.24,2.11$, and 2.18 , respectively.

Task instructions produced a significant effect during habituation despite the fact that the task for which the students in the three experimental groups had been instructed to perform had not yet occurred. Students were differentially orienting to the words, at least in the pedal-press and associate groups, in anticipation of "light." Of particular interest is the fact that the effect was not present at the outset, but developed over successive words. There was no significant difference in the GSR-OR evoked in the different groups by the first word. Unless very explicit instructions are given (Pendery \& Maltzman, 1977), the individual difference effect or the effect of instructions develops gradually over trials. Such differences reflect, we assume, the establishment of distinctive modes of thinking and orienting. The lack of difference in the GSR-OR to the first word and the subsequent development of such a difference among the groups suggests that the initial word evokes a relatively involuntary OR. It is not affected by the kinds of instructions offered, because the first word remained a relatively unpredictable stimulus. The GSR-ORs evoked by subsequent words in the habituation series are affected by instructions because the class of stimuli is increasingly predictable. Complex cortical processes have an opportunity to come into play and modulate the ORs induced by the stimuli. The initial OR to the first unpredictable stimulus is not as susceptible to these predisposing cortical processes as ORs to subsequent stimuli.

Luria (1973) also distinguishes between voluntary and involuntary ORs in a manner much the same as the present one. He provides evidence indicating that the involuntary OR usually evoked by nonsignificant stimuli, in contrast to the voluntary OR evoked by significant stimuli, differ in their morphology and physiology. Brain lesions in different loci differentially affect the two kinds of OR.

Log conductance base level was also analyzed during habituation. The only significant betweensubjects effect obtained was attributable to OR level $\left[\mathrm{F}(1,128)=71.12, \mathrm{MS}_{\mathrm{B}}=1.114\right]$. Trials were $\mathrm{a}$ significant within-subject factor $[F(19,2432)=27.89$, $\mathrm{MSW}=.214]$. Analysis of orthogonal polynomials indicated that the conductance level of the groups declined at different rates $\left[\mathrm{F}(3,128)=7.30, \mathrm{MS}_{\mathrm{B}}\right.$ $=.139$ ]. Mean L scores obtained from the response trends for the four groups, associate, pedal-press, count, and control, were $-.03,-.01,-.04$, and -.13 , respectively. Rate of decline in conductance level was greater in the control than in the experimental groups. A series of $t$ tests indicated that each of the experimental groups differed significantly from the control group in terms of their mean L scores. There were no significant differences among the experimental groups, suggesting that the different kinds of task instructions had a similar effect of retarding the decline in tonic alertness or orienting. 


\section{Training}

Table 1 presents the mean magnitude of the GSRs evoked by the critical word "light" and the immediately preceding $\left(C_{1}\right)$ and following $\left(C_{2}\right)$ control words for the 14 training trials in each of the four groups, associate, pedal-press, count, and control. Response differentiation was obtained in each of the groups except control. The pedal-press group clearly yielded the largest GSRs to the critical word "light." While the count and associate groups did not differ from each other appreciably, each was superior to the control group.

Analyses of variance and orthogonal polynomials of the main effects and response trends over the training trials substantiate the impression of differences shown in Table 1. Significant between-subjects factors were OR level $[\mathrm{F}(1,32)=48.64]$, and Groups $\left[\mathrm{F}(3,132)=8.31, \mathrm{MS}_{\mathrm{B}}=8.360\right]$. More importantly, there were a number of significant within-subject effects: Trials $\left[\mathrm{F}(13,1716)=6.96, \mathrm{MS}_{\mathrm{W}}=.341\right]$, Words $\left[\mathrm{F}(2,264)=167.7, \mathrm{MS}_{\mathrm{W} 2}=.968\right]$, Word by Trials $\left[\mathrm{F}(26,3434)=3.17, \mathrm{MS}_{\mathrm{W} 3}=.260\right]$, Groups by Trials $\left[\mathrm{F}(29,1716)=2.39, \mathrm{MS}_{\mathrm{W} 1}=.341\right]$, OR by Words $\left[\mathrm{F}(2,264)=12.08, \mathrm{MS}_{\mathrm{W} 2}=.968\right]$, and Groups by Words $\left[\mathrm{F}(6,264)=24.50, \mathrm{MS}_{\mathrm{W} 2}=.968\right]$, the interaction of primary interest. Analysis of orthogonal polynomials indicated a significant decreasing overall response trend, as well as a significant overall difference in trends for Groups and for the Groups by Words interaction.

Analyses were also conducted on the GSRs evoked by "light" in each of the groups, disregarding the responses to the control words. In order to simplify programming this analysis, two participants were randomly discarded from the associate and count groups, leaving an equal $n, 34$, in each of the four groups. Groups was significantly different $[F(3,128)$ $=20.78]$ and $O R$ was a significant effect $[F(1,128)$ $\left.=46.97, \mathrm{MS}_{\mathrm{B}}=5.028\right]$. Trials and the Group by Trials interaction was also significant $[\mathrm{F}(13,1664)=$ 10.42 and $\mathrm{F}(39,1664)=3.67, \mathrm{MS}_{\mathrm{W}}=.268$ ]. Individual comparisons by $t$ test indicated that each of the three experimental groups produced significantly larger GSRs to the critical word than did the control group. The pedal-press group was also significantly

Table 1

Mean Magnitude of the GSRs Evoked by the Critical Word "Light" and the Immediately Preceding $\left(C_{1}\right)$ and Following $\left(C_{2}\right)$ Control Words During Training for Each of the Four Groups, Associate, Pedal Press, Count, and Control

\begin{tabular}{lccc}
\hline & \multicolumn{3}{c}{ Words } \\
\cline { 2 - 4 } Group & $\mathrm{C}_{1}$ & LIGHT & $\mathrm{C}_{2}$ \\
\hline Associate & .42 & .85 & .49 \\
Pedal Press & .57 & 1.57 & .55 \\
Count & .31 & .83 & .35 \\
Control & .31 & .44 & .40 \\
\hline
\end{tabular}

more responsive to "light" than was the count group. No other differences among the experimental groups were statistically significant.

Analysis of orthogonal polynomials yielded a significant main effect, indicating that the overall trend was a decline in responsivity, and a difference in Group trends $[\mathrm{F}(1,128)=37.64$ and $\mathrm{F}(3,128)=$ 10.45 , respectively; $\mathrm{MS}_{\mathrm{B}}=.649$ ]. The mean L scores obtained by the associate, pedal-press, count, and control groups were $-.74,-.79,-.34$, and .17 , respectively. Each of the experimental groups showed a declining trend, whereas the control group showed a slight increase in their response trend to "light", during the course of the training phase. Groups also showed a significant difference in the quadratic components of their trends.

Results from the training session indicate that assigning significance to a stimulus by instructing students to overtly or covertly respond retards habituation, but habituation nevertheless occurs. The slight increase in responsivity manifest by the control group could be a chance effect since it is not great. However, verbal protocols obtained from all students after completion of the experiment suggest another possibility. The increase in responsivity may be a reflection of frustration or annoyance reported by the students because of the boring and repetitive nature of the task. Increased responsivity in the control group may therefore be a reflection of overextinction (Sokolov, 1963).

Analyses of conductance level during training yielded results somewhat comparable to those obtained during habituation. The only between-subjects effect was yielded by OR level $[F(1,128)=46.75$, $\left.\mathrm{MS}_{\mathrm{B}}=1.019\right]$. Trials yielded a significant withinsubject effect $\left[\mathrm{F}(13,1664)=35.42, \mathrm{MS}_{\mathrm{W}}=.419\right]$. In addition to an overall significant decline in conductance level, the trends for Groups differed significantly as reflected in a Groups by Trials interaction and a significant difference in the linear components of the trends, as shown by the analysis of orthogonal polynomials $\left[\mathrm{F}(3,128)=2.75, \mathrm{MS}_{\mathrm{B}}=\right.$ .302]. The mean $L$ scores for the pedal-press and count groups displayed a significantly slower rate of decline than the associate and control groups, which did not differ.

\section{Conclusions}

Findings obtained in this study indicate that the significance of a cue induced by instructing students to covertly respond augments the GSR-ORs evoked by this cue in a manner similar to the effect of an overt response. Selective orienting can be induced by task instructions requiring covert as well as overt differential responding. In addition, these task instructions increase orienting to all words prior to the advent of the specific task. Clearly, then, elicita- 
tion of the GSR-OR need not be solely a consequence of stimulus change, but is modulated in great degree by the disposition of the individual.

Absence of a difference among the groups in their GSR-OR to the first word in habituation, as contrasted with the subsequent development of differences in habituation as well as training, suggests that somewhat different processes are affecting the GSR-OR to an initial unpredictable stimulus, as contrasted to later relatively predictable stimuli. This difference is one basis for distinguishing between what may be called involuntary, as contrasted with voluntary, ORs.

Selective orienting or attention was produced in the present experiment in that nonsignificant, irrelevant words yielded reliably smaller GSR-ORs than the signal word. The responses to "light" were significantly greater than the responses to the immediately preceding and following control words. There was no evidence that the selective OR to the critical signal word produced inhibition or facilitation of the OR to the irrelevant words. The control group that sat quietly and listened showed ORs of the same magnitude to the irrelevant words as did the experimental groups even though the latter showed disproportionately greater GSR-ORs to the critical word. These results therefore demonstrate selective attention or selective phasic ORs that cannot be accounted for in terms of increases in nonspecific arousal (Näätänen, 1967), a criticism of earlier evoked potential studies of selective attention. The criticism that what appears to be selective attention is actually a consequence of nonspecific arousal effect was circumvented by imbedding the critical word among noncritical words in a relatively unpredictable manner and recording the responses to the noncritical as well as the critical words in control as well as experimental groups. In this connection, it is important to note that implicit activity, such as counting as well as overt button pressing, has been used in selective attention studies with averaged evoked potentials as the measure of attention (e.g., Donchin \& Cohen, 1967). That nonspecific arousal effects are also induced by task instructions was indicated by the slower rate of habituation of conductance level by experimental groups as compared to the control group in both habituation and training. But the selective GSR-ORs to the critical word interspersed among noncritical words indicates that nonspecific arousal or tonic ORs cannot be the sole basis for selective attention or set as Floru (1975) has suggested.

The present results showing that implicit activity, free associating and counting, induce GSRs is relevant to the interpretation of GSR experiments that have employed so-called spontaneous GSRs as an indication of individual differences in personality, emotionality, or conditioning (Bernstein, 1973; Johnson, 1963; Mundy-Castle \& McKiever, 1953). These GSRs have been called "spontaneous" because they occur in the absence of any external stimulus change introduced by the experimenter. They are often measured during rest periods prior to the start of an experiment or periods during the course of an experiment free of external stimulus changes. Rather than directly reflecting anxiety, emotionality, or peripheral lability, the results of training and habituation suggest that these spontaneous GSRs may reflect responses induced as a consequence of the participants' thinking or orienting to internal cues. Bernstein (1973) has reached a similar conclusion from different kinds of data. They may be generated by the integrative cortical activity of the participants and in this sense represent voluntary GSR-ORs. Individual differences in spontaneous GSRs may therefore reflect individual differences in styles of thinking rather than anxiety or emotionality in some more direct sense. It is obvious, at least, that an autonomic measure such as the GSR cannot be equated in any simple manner with a theoretical concept such as emotionality, anxiety, or nonspecific arousal.

\section{REFERENCE NOTE}

1. Russell, W. A., \& Jenkins, J. J. The complete Minnesota norms for responses to 100 words from the Kent-Rosanoff Word Association Test. Technical Report No. 11, Contract No. N8 ONR-6616, Office of Naval Research and University of Minnesota, 1954.

\section{REFERENCES}

Bernstein, A. S. Electrodermal lability and the OR: Reply to O'Gorman and further exposition of the "significance hypothesis." Australian Journal of Psychology, 1973, 25, 147-154.

Donchin, E., \& CohEn, L. Averaged evoked potentials and intramodality selective attention. Electroencephalography and Clinical Neurophysiology, 1967, 22, 537-546.

FLORU, R. Psychophysiological investigations on attention. In E. N. Sokolov \& O. S. Vinogradova (Eds.), Neuronal mechanisms of the orienting reflex. Hillsdale, N.J: Erlbaum, 1975.

JoHnson, L. D. Some attributes of spontaneous electrodermal activity. Journal of Comparative and Physiological Psychology, 1963, 56, 415-422.

LURIA, A. R. The working brain. New York: Basic Books, 1973.

LuRIA, A. R., \& Vinogradova, O. S. An objective investigation of the dynamics of semantic systems. British Journal of Psychology, 1959, 50, 89-105.

Maltzman, I. The orienting reflex and thinking as determiners of conditioning and generalization to words. In $\mathrm{H}$. $\mathrm{H}$. Kendler \& J. T. Spence (Eds.), Essays in neobehaviorism: A memorial volume to Kenneth $W$. Spence. New York: Appleton-CenturyCrofts, 1971.

Maltzman, I., Gould, J., Barnett, O. J., Raskin, D. C., \& WolfF, C. Classically conditioning components of the orienting reflex to words using an innocuous and a noxious UCS under different CS-UCS intervals. Journal of Experimental Psychology: General, 1977, 106, 185-212.

Maltzman, I., \& Langdon, B. Semantic generalization of the GSR as a function of semantic distance or the orienting reflex. Journal of Experimental Psychology, 1969, 80, 289-294. 
Maltzman, I., Langdon, B., Pendery, M., \& Wolff, C. The GSR orienting reflex and semantic conditioning and generalization with different unconditioned stimuli. Journal of Experimental Psychology: General. 1977, 106, 141-171.

Maltzman, I., \& Raskin, D. C. Effects of individual differences in the orienting reflex on conditioning and complex processes. Journal of Experimental Research in Personality, 1965, 1, 1-16. MedNick, M. T. Mediated generalization and the incubation effect as a function of manifest anxiety. Journal of Abnormal and Social Psychology, 1957, 55, 315-321.

Mundy-Castle, A. C., \& McKiever, B. L. The psychophysiological significance of the galvanic skin response. Journal of Experimental Psychology, 1953, 46, 15-24.
NÄ̈TXNEN, R. Selective attention and evoked potentials. Helsinki: Suomalainen Tiedeakatemia, 1967.

Pendery, M., \& Maltzman, I. Instructions and the orienting reflex in "semantic conditioning" of the GSR in an innocuous situation. Journal of Experimental Psychology: General, 1977, 106, 120-140.

Sokolov, E. N. Perception and the conditioned reflex. New York: Macmillan, 1963.

(Received for publication February 1, 1978; revision accepted January $9,1979$. 